\title{
Solutions of Navier-Stokes Equations with Coriolis Force in the Rotational Framework
}

Nghiệm của các phương trình Navier-Stokes với lực Coriolis trong môi trường khung quay

\section{Vu Thi Bich Tuyen}

Hanoi University of Science and Technology, Hanoi, Vietnam

Email: tuyen.vuthibich@hust.edu.vn

\begin{abstract}
In this article, for $0 \leq m<\infty$ and the index vectors $q=\left(q_{1}, q_{2}, q_{3}\right), r=\left(r_{1}, r_{2}, r_{3}\right)$ where $1 \leq q_{i} \leq \infty, 1<r_{i}<\infty$ and $1 \leq i \leq 3$, we study new results of Navier-Stokes equations with Coriolis force in the rotational framework in mixed-norm Sobolev-Lorentz spaces $\dot{H}^{m, r, q}\left(R^{3}\right)$, which are more general than the classical Sobolev spaces. We prove the existence and uniqueness of solutions to the Navier-Stokes equations (NSE) under Coriolis force in the spaces $L^{\infty}\left([0, T] ; \dot{H}^{m, r, q}\right)$ by using topological arguments, the fixed point argument and interpolation inequalities. We have achieved new results compared to previous research in the Navier-Stokes problems.
\end{abstract}

Keywords: Navier-stokes equations, coriolis force, rotation framework.

Tóm tắt

Trong bài báo này, cho $0 \leq m<\infty$ và các véctơ chỉ số $q=\left(q_{1}, q_{2}, q_{3}\right), r=\left(r_{1}, r_{2}, r_{3}\right)$, với $1 \leq q_{i} \leq \infty, 1<r_{i}<\infty$ và $1 \leq i \leq 3$, chúng tôi nghiên cứu những kết quả mới của các phương trình Navier-Stokes với lực ngoài Coriolis trong không gian chuẩn kết hợp Sobolev-Lorentz $\dot{H}^{m, r, q}\left(R^{3}\right)$, không gian tổng quát hơn không gian Sobolev cổ điển đã biết. Chúng tôi chứng minh sự tồn tại và tính duy nhất nghiệm của các phương trình Navier-Stokes (NSE) dưới tác động của lực Coriolis trong không gian $L^{\infty}\left([0, T] ; \dot{H}^{m, r, q}\right)$ bằng cách sử dụng tính chất các không gian, lý thuyết điểm bất động và các bất đẳng thức nội suy. Chúng tôi đã đạt được những kết quả mới so với các nghiên cứu trước đó về các bài toán Navier-Stokes.

Từ khóa: Các phương trình Navier-stokes, lực Coriolis, khung quay.

\section{Introduction}

The Coriolis force arises in almost all of the models of meteorology and geophysics dealing with large-scale phenomena. If we look around to see what is happening, we realize that the rotation leads to many interesting phenomena, this effect is substantial: the climate is affected and complicated because the rotation changes the flow of air heated by the sun. Typhoons and their motion are another phenomenon resulting from the effect of rotation of Earth. The flows of the ocean can be affected by the rotation of Earth as well. Changing the motion of moving objects of fluids such as air and ocean illustrated above is caused by force. This force on all moving bodies is called Coriolis force. Almost all of the fluids following on Earth can be affected, so researching the Coriolis effects in Navier-Stokes equations are very important. We consider Navier-Stokes equations in the rational framework with Coriolis force in $R^{3}$. For the recent results concerning periodic solutions for the NavierStokes equations in the rotational framework, we refer to the work of Kozono, Mashiko and Takada [1]. They proved the existence of unique mild periodic solution with suitable smallness assumptions on forces.

The problems of mild solutions to the NavierStokes equations and the problems concerning NavierStokes equations have a long history, first results on constructing mild solutions for the Navier-Stokes equations in the Sobolev spaces $H^{s}\left(R^{3}\right)$, go back to Kato and Fujita [2].

In 1995, using the method of the bilinear operator, Cannone [3] proved the existence of mild solutions in $\dot{H}^{m}\left(R^{3}\right)$, Giga [4] continued to establish

ISSN: 2734-9381

https://doi.org/10.51316/jst.153.etsd.2021.31.5.8

Received: August 14, 2019; accepted: August 26, 2021 
the mild solutions with value in $L^{q}\left(R^{3}\right)$ and Cannone and Meyer [3,5] studied the existence of mild solutions with initial data in the space $L^{\infty}$. Base on the Besov spaces on a bounded domain in $R^{3}$, the regularity and uniqueness conditions for weak solutions of NSE were established in [6]. Kato and Ponce [7] showed that the Navier-Stokes problems are well-posed when the initial data in the homogeneous Sobolev spaces.

Recently, D.Q. Khai and N.M. Tri considered the mild solutions of Navier-Stokes in mixed-norm Sobolev-Lorentz spaces and Sobolev-Fourier-Lorentz spaces, as in $[8,9]$

This paper approaches to the methods to solve the problems of existence and uniqueness of the solution of the Navier-Stokes equations with Coriolis forces in new spaces $L^{\infty}\left([0, \mathrm{~T}] ; \dot{H}^{m, r, q}\right)$. It develops the results in [8] which shows the existence and uniqueness of the solutions of Navier-Stokes equations in the same spaces.

We consider the Navier-Stokes equations in the rotational framework which are given by the equation

$$
\left\{\begin{array}{c}
u_{t}+(u . \nabla) u-\Delta u+\nabla p=\omega e_{3} \times u+\operatorname{div} F \\
\nabla . u=0 \\
u(0)=u_{0}
\end{array}\right.
$$

where $\omega$ is the speech of rotation and $e_{3}$-the unit vector in $x_{3}$-direction. The unknown quantities are the velocity $u(t, x)=\left(u_{1}(t, x), u_{2}(t, x), u_{3}(t, x)\right)$ of the fluid element at time $t$ and position $x$ with the pressure $p(t, x)$.

Apply the Helmhotz projection P we obtain

$$
\left\{\begin{array}{c}
u_{t}-P \Delta u=P\left(\omega e_{3} \times u\right)+P \operatorname{div}(-u u)+g \\
\nabla \cdot u=0 \\
u(0)=u_{0}, g=\operatorname{div} F
\end{array}\right.
$$

We first define the definition of mild solution to (2). We mean the function $u(t)$ satisfying

$$
u(t)=e^{t \Delta} u_{0}+\int_{0}^{t} e^{(t-\tau) \Delta} P\left[\nabla \cdot(-u u)+g+\omega e_{3} \times u(\tau)\right] d \tau
$$

We note the mixed-norm Sobolev -Lorentz spaces $\dot{H}^{m, r, q}$ is considering as the space $(\sqrt{-\Delta})^{-m} L^{r, q}$, equipped with the norm $\|u\|_{\dot{H}^{m, r, q}}=\left\|(\sqrt{-\Delta})^{m} u\right\|_{L^{r, q}}$.

\section{Main results}

We recall the following results

Lemma 2.1. Let $q=\left(q_{1}, q_{2}, q_{3}\right), r=\left(r_{1}, r_{2}, r_{3}\right), m \geq 0$ and $0<T<\infty$ be such that

$$
\begin{aligned}
& \frac{1}{q_{1}}+\frac{1}{q_{2}}+\frac{1}{q_{3}}-1<m<\frac{\frac{1}{q_{1}}+\frac{1}{q_{2}}+\frac{1}{q_{3}}}{2}, 1 \leq q_{i} \leq \infty \\
& 2<\frac{q_{i}}{1-\frac{m}{\frac{1}{q_{1}}+\frac{1}{q_{2}}+\frac{1}{q_{3}}}}<\infty, 1 \leq i \leq 3
\end{aligned}
$$

Then the bilinear operator

$$
B(u, v)(t)=\int_{0}^{t} e^{(t-\tau) \Delta} P[\nabla \cdot(-u v) d \tau
$$

is continuous from $L^{\infty}\left([0, \mathrm{~T}] ; \dot{H}^{m, r, q}\right) \times L^{\infty}([0, \mathrm{~T}]$; $\left.\dot{H}^{m, r, q}\right)$ to $L^{\infty}\left([0, \mathrm{~T}] ; \dot{H}^{m, r, q}\right)$ and we have the the enequality

$$
\begin{aligned}
& \|B(u, v)(t)\|_{L^{\infty}\left([0, \mathrm{~T}] ; \dot{H}^{m, r, q}\right)} \\
& \leq T \frac{m+1-\left(\frac{1}{q_{1}}+\frac{1}{q_{2}}+\frac{1}{q_{3}}\right)}{2}\|u\|_{L^{\infty}\left([0, \mathrm{~T}] ; \dot{H}^{m, r, q}\right)} \times\|v\|_{L^{\infty}\left([0, \mathrm{~T}] ; \dot{H}^{m, r, q}\right)} .
\end{aligned}
$$

Proof. This lemma is obtained from lemma 3 in [8].

Lemma 2.2. If $u \in \dot{H}^{m, r, q}$ then

$$
\left\|e^{t \Delta} u\right\|_{L^{\infty}\left([0, \mathrm{~T}] ; \dot{H}^{m, r, q}\right)} \leq\|u\|_{L^{\infty}\left([0, \mathrm{~T}] ; \dot{H}^{m, r, q}\right)}
$$

Proof. See lemma 4 in [8].

Lemma 2.3. Let $P$ is the Helmholtz projection onto the divergence-free fields then $\mathrm{P}$ is continuous from $L^{r, q}$ to $L^{r, q}, 1<r<\infty, 1 \leq q \leq \infty$.

Proof. The operator P in [8] is defined by the Riesz transforms

$$
(P f)_{j}=f_{j}+\sum_{1 \leq k \leq 3} R_{j} R_{k} f_{k}
$$

and $R_{j}$ is bounded from $L^{p}$ to $L^{p}, 1<p<\infty$ see in ([10], theorem 6.8 and example, p.54) then $\mathrm{P}$ is also bounded from $L^{p}$ to $L^{p}, 1<p<\infty$. In addition, we have the interpolation relation

$$
\left(L^{p_{1}}, L^{p_{2}}\right)_{\theta, q}=L^{r, q}, 1<p_{1}<r<p_{2}, 1 \leq q \leq \infty
$$


Using the interpolation theory, we obtain that the operator $\mathrm{P}$ is bounded from $L^{r, q}$ to $L^{r, q}, 1<r<\infty, 1 \leq q \leq \infty$.

Therefore, based on linearity property, $\mathrm{P}$ is continuous from $L^{r, q}$ to $L^{r, q}$ and the proof is completed.

Lemma 2.4. If $u \in \dot{H}^{m, r, q}$ then $\forall 0<t \leq T$

$$
\left\|\int_{0}^{t} e^{(t-\tau) \Delta} P u(\tau) d \tau\right\|_{L^{\infty}\left([0, \mathrm{~T}] ; \dot{H}^{m, r, q}\right)} \leq \mathrm{T}\|u\|_{L^{\infty}\left([0, \mathrm{~T}] ; \dot{H}^{m, r, q}\right)} .
$$

Proof. We have

$$
\begin{aligned}
& \left\|\int_{0}^{t} e^{(t-\tau) \Delta} P u(\tau) d \tau\right\|_{\dot{H}^{m, r, q}} \\
& =\left\|\int_{0}^{t} e^{(t-\tau) \Delta}(\sqrt{-\Delta})^{m} P u(\tau) d \tau\right\|_{L^{r, q}} \\
& =\left\|\int_{0}^{t} \frac{1}{[4 \pi(t-\tau)]^{\frac{3}{2}}} \int_{R^{3}} e^{\frac{-|\theta|^{2}}{4(t-\tau)}}(\sqrt{-\Delta})^{m} P u(.-\theta) d \theta d \tau\right\|_{L^{r, q}} \\
& \leq \int_{0}^{t} \frac{1}{[4 \pi(t-\tau)]^{3 / 2}} \int_{R^{3}}^{\frac{-|\theta|^{2}}{4(t-\tau)}}\|P u(\tau, .-\theta)\|_{H^{m, r, q}} d \theta d \tau \\
& \leq\|P u\|_{L^{\infty}\left([0, \mathrm{~T}] ; \dot{H}^{m, r, q}\right)} \int_{0}^{t} \frac{1}{[4 \pi(t-\tau)]^{\frac{3}{2}}} \int_{R^{3}} e^{\frac{-|\theta|^{2}}{4(t-\tau)}} d \theta d \tau \\
& \leq \mathrm{T}\|u\|_{L^{\infty}\left([0, \mathrm{~T}] ; \dot{H}^{m, r, q}\right)} \cdot
\end{aligned}
$$

Therefore, we obtain the lemma.

\section{Theorem 2.1.}

Let

$$
\begin{gathered}
q=\left(q_{1}, q_{2}, q_{3}\right), r=\left(r_{1}, r_{2}, r_{3}\right), m \geq 0,\|g\|_{L^{\infty}\left([0, \mathrm{~T}] ; \dot{H}^{m, r, q}\right)}, \\
\left\|u_{0}\right\|_{\dot{H}^{m, r, q}}
\end{gathered}
$$

be small enough and $0<T<\infty$ be such that

$$
\begin{gathered}
\frac{1}{q_{1}}+\frac{1}{q_{2}}+\frac{1}{q_{3}}-1<m<\frac{\frac{1}{q_{1}}+\frac{1}{q_{2}}+\frac{1}{q_{3}}}{2}, 1<r_{i}<\infty \\
2<\frac{q_{i}}{1-\frac{m}{\frac{1}{q_{1}}+\frac{1}{q_{2}}+\frac{1}{q_{3}}}}<\infty, 1 \leq q_{i} \leq \infty, 1 \leq i \leq 3
\end{gathered}
$$

Then the equation (2) has a unique mild solution $u$ in a small ball of $L^{\infty}\left([0, \mathrm{~T}] ; \dot{H}^{m, r, q}\right)$.

Proof. Consider the ball

$$
B_{\varepsilon}=\left\{v \in L^{\infty}\left([0, \mathrm{~T}] ; \dot{H}^{m, r, q}\right):\|v\|_{L^{\infty}\left([0, \mathrm{~T}] ; \dot{H}^{m, r, q}\right)} \leq \varepsilon\right\}
$$

Let $\varphi$ be the transformation given as $\varphi(v)=u$ where $u$ is the unique mild solution to equation $u_{t}-\mathrm{P} \Delta \mathrm{u}=\mathrm{P}\left(\omega e_{3} \times v\right)+P \operatorname{div}(-v v)+g$ (see theorem 6 in [8])

$$
u(t)=e^{t \Delta} u_{0}+\int_{0}^{t} e^{(t-\tau) \Delta} P\left[\nabla .(-v v)+g+\omega e_{3} \times v(\tau)\right] d \tau
$$

We will prove that if $\varepsilon$ and $\|g\|_{L^{\infty}\left([0, \mathrm{~T}] ; \dot{H}^{m, r, q}\right)}$ are small enough then the transformation $\varphi$ acts from $B_{\varepsilon}$ is a contraction. To do this, we estimate

$$
\begin{aligned}
& \|u(t)\|_{L^{\infty}\left([0, \mathrm{~T}] ; \dot{H}^{m, r, q}\right)} \leq\left\|e^{t \Delta} v_{0}\right\|_{L^{\infty}\left([0, \mathrm{~T}] ; \dot{H}^{m, r, q}\right)} \\
& +\|B(v, v)\|_{L^{\infty}\left([0, \mathrm{~T}] ; \dot{H}^{m, r, q}\right)} \\
& +\left\|\int_{0}^{t} e^{(t-\tau) \Delta} P\left[g+\omega e_{3} \times v(\tau)\right] d \tau\right\|_{L^{\infty}\left([0, \mathrm{~T}] ; \dot{H}^{m, r, q}\right)} \\
& \leq\left\|v_{0}\right\|_{H^{m, r, q}}+T \frac{\frac{m+1-\left(\frac{1}{q_{1}}+\frac{1}{q_{2}}+\frac{1}{q_{3}}\right)}{2}}{\|v\|_{L^{\infty}\left([0, \mathrm{~T}] ; \dot{H}^{m, r, q}\right)}} \\
& \times\|v\|_{L^{\infty}\left([0, \mathrm{~T}] ; \dot{H}^{m, r, q}\right)}+T\left\|g+\omega e_{3} \times v\right\|_{L^{\infty}([0, \mathrm{~T}] ;)} \\
& \leq \varepsilon / 2+T \frac{\frac{m+1-\left(\frac{1}{q_{1}}+\frac{1}{q_{2}}+\frac{1}{q_{3}}\right)}{2}}{2} \varepsilon^{2}+T\left(\|g\|_{L^{\infty}\left([0, \mathrm{~T}] ; \dot{H}^{m, r, q}\right)}+\varepsilon\right) \\
& \leq \varepsilon \\
& -v v+v^{*} v^{*}=\left(v^{*}-v\right) v+v^{*}\left(v^{*}-v\right) \\
& \varphi(v)-\varphi\left(v^{*}\right)=\int_{0}^{t} e^{(t-\tau) \Delta} P \nabla \cdot\left\{\left[\left(v^{*}-v\right) v+v^{*}\left(v^{*}-v\right)\right]\right. \\
& \left.+\omega e_{3} \times\left(v-v^{*}\right)(\tau)\right\} d \tau
\end{aligned}
$$

Therefore,

$\left\|\varphi(v)-\varphi\left(v^{*}\right)\right\|_{L^{\infty}\left([0, \mathrm{~T}] ; \dot{H}^{m, r, q}\right)}$ 


$$
\begin{aligned}
& \leq \| \int_{0}^{t} e^{(t-\tau) \Delta} P \nabla \cdot\left\{\left[\left(v^{*}-v\right) v+v^{*}\left(v^{*}-v\right)\right]\right. \\
& \left.\quad+\omega e_{3} \times\left(v-v^{*}\right)(\tau)\right\} d \tau \|_{L^{\infty}\left([0, \mathrm{~T}] ; \dot{H}^{m, r, q}\right)}\left[\|v\|_{L^{\infty}\left([0, \mathrm{~T}] ; \dot{H}^{m, r, q}\right)}+\left\|v^{*}\right\|_{L^{\infty}\left([0, \mathrm{~T}] ; \dot{H}^{m, r, q}\right)}\right] \\
& \leq T \frac{m+1-\left(\frac{1}{q_{1}}+\frac{1}{q_{2}}+\frac{1}{q_{3}}\right)}{2}\left[\left\|v-v^{*}\right\|_{L^{\infty}\left([0, \mathrm{~T}] ; \dot{H}^{m, r, q}\right)}\right. \\
& \left.\leq 2 \varepsilon T \frac{m+1-\left(\frac{1}{q_{1}}+\frac{1}{q_{2}}+\frac{1}{q_{3}}\right)}{2}\right)\left\|v-v^{*}\right\|_{L^{\infty}\left([0, \mathrm{~T}] ; \dot{H}^{m, r, q}\right)} \\
& <\left\|v-v^{*}\right\|_{L^{\infty}\left([0, \mathrm{~T}] ; \dot{H}^{m, r, q}\right)} \text { if } \mathrm{T} \text { is small enough. }
\end{aligned}
$$

It implies that that $\varphi$ is a contraction and the equation (2) has unique solution in this ball.

Therefore, the equation (1) has the unique solution in the ball of $L^{\infty}\left([0, \mathrm{~T}] ; \dot{H}^{m, r, q}\right)$ under some conditions and we obtain the main results of this paper. In addition, we also continue to study this solution of the global problems in the same space.

\section{Conclusion}

Our obtained results are considered as an impetus to solve the problems of Navier-Stokes equations in the new Sobolev space which has been presented in [8].

\section{Acknowledgments}

This research is funded by the Hanoi University of Science and Technology (HUST) under project number T2018-PC-122.

\section{References}

[1] H. Kozono, M. Mashiko, R. Takada, Existence of periodic solutions and their asymptotic stability to the
Navier-Stokes equations with coriolis force, J. Evol. Equa. 14, (2014) 565-601.

[2] T. Kato, H. Fujita, On the non-stationary NavierStokes system, Rend. Semin. Mat. Univ. Padova 32 (1962) 243-260

[3] M. Cannone, Y. Meyer, Littlewood-Paley decomposition and the Navier-Stokes equations, Methods Appl. Anal. 2 (1995) 307-319.

[4] Y. Giga, Solutions of semilinear parabolic equations in $L^{p}$ and regularity of weak solutions of the NavierStokes system, J. Differential Equations 62 (1986) 186-212.

[5] M. Cannone, Ondeletters, Paraproduits et NavierStokes, Diderot Editeur, Paris, 1995.

[6] R. Farwig, H. Sohr, W. Varnhorn, Besov space regurity conditions for weak solutions of the NavierStokes equations, J. Math. Fluid Mech. 16 (2014) 307320 .

[7] T. Kato, G. Ponce, The Navier-Stokes equations with weak initial data, Int. Math. Res. Not. 10. (1994) 435444.

[8] D.Q. Khai, N.M. Tri, Solutions in mixed-norm Sobolev-Lorentz spaces to the initial value problem for the Navier-Stokes equations, J. Math. Anal. Appl. 417 (2014) 814-833.

[9] D.Q. Khai, N.M. Tri, Well-posedness for the NavierStokes with data in homogeneous Sobolev-Lorentz spaces, Nonlinear Analysis 149 (2017) 130-145.

[10] P.G. Lemarie-Rieusset, Recent developments in the navier-stokes problem, Chapman and hall/CRC Research Notes in Mathematics, vol. 431, Chapman and Hall/CRC, Boca Raton, FL, (2002).

[11] Sunggeun Lee, Shyn-Kun Ryi, Hankwon Lim; Solutions of Navier-Stokes equation with coriolis force, Advances in Mathematical Physics, volume 2017.

[12] J. Bergh, L. Löfström, Interpolation theory, Spaces. Springer, Berlin (1976) 